\title{
Improving face recognition by elman neural network using curvelet transform and HSI color space
}

\author{
Ahmed S. Abdullah ${ }^{1}$, Majida Ali Abed ${ }^{2}$, Israa Al_Barazanchi ${ }^{3}$ \\ ${ }^{1,2}$ College of Computers Sciences \& Mathematics, Tikrit University \\ ${ }^{3}$ Baghdad College of Economic Sciences University
}

\section{Article Info}

Received Jan 17, 2019

\section{Keyword:}

face recognition, image processing, color space, neural network, curve let transform.

\begin{abstract}
In this paper, a suggested algorithm was proposed to increase the efficiency of the Elman neural algorithm in face recognition. The proposed algorithm was studied on the images of 20 students from the Department of Computer Science, Tikrit University. First step creates dataset of faces, second step convert color space to HSI and using saturation layer, image decomposition using curvelet transform, feature extraction using Principle component analysis, and final step face recognition using Elman neural network. after applying proposed algorithm, the rate of face recognition $94 \%$.
\end{abstract}

\section{Corresponding Author:}

\author{
Ahmed S. Abdullah ${ }^{1}$, \\ College of Computers Sciences \& Mathematics \\ Tikrit University, Tikrit, Iraq \\ Email: Albashaahmed1985@gmail.com.
}

\section{Introduction}

Pattern recognition is the process of using computer software to classify things based on some of the characteristics that make them different from other with less error [1-3]. One of the most important topics that began to take a wide area concerning, Pattern recognition is individual's recognition as there are characteristics can be used to distinguish from one person to another [4]. for instance, the behavioral characteristics such as the method of writing or the way of making a signature, we can also make use of qualities such as the tone of voice, facial feature, iris of eye or the fingerprint [5]. face recognition is one of the most widely used application in many areas where it can be used an alternative to a password or for criminals identification. Several studies conducted have been conducted on the process of distinguishing faces [6-7]. in this research presented a proposed method to face recognition using curvelet transform, neural network and saturation layer in HSI color space.

\section{Related work}

Mary (2011) presented face recognition paper used wavelet transform , principle component analysis, skin color and Back-propagation N.N, Where the work was divided into several steps The first stage is the stage of detection the face and then disassemble the image using the wavelet transformations after the properties are obtained using (PCA) after which the application of the Backpropagation neural network , The neural network is designed of three layers: the input layer contains 40 neurons and the output layer is 10 neurons and the hidden layer is 20 neurons[8]. 
Al-Hamdani (2012) presented face recognition paper used PCA and Euclidean Distance Where the method is divided into several steps The first step is the process of detection of the face and then remove the noise from the image after being used principle component analysis to features extraction and the final step using Euclidean distance [9]

Miry(2013) presented face recognition paper used wavelet transform and PCA with Back propagation neural network. the level of image decomposition was measured using wavelet transformations. The image was disassembled using three levels. The study showed that the best level of disassembly is the third level [10].

Many researches (2014) presented paper to enhanced principle component analysis method,by dividing the image used in the process of training the network into categories of each category of a single person measured in all its different specifications such as the use of glasses or change hair styles, so PCA applies to the number of people used in databases [11].

Ali (2015) Presented paper based on vector conversions for image decomposition was adopted in the research on 5 levels of disassembly after which using probabilistic N.N after their using support vector machine, the method applied was applied to different types of images. The method was applied to the original image. This was added to the noise of salt and pepper. The method was also studied adding Gaussian noise, the median filter was applied to the image and then the proposed algorithm was applied [12], after that many papers presented to distinguish and identify face recognition using wavelet transformations and PCA [13-16]. In this paper used curvelet transform and HSI color space the result of propose algorithm batter than related method.

\section{Curvelet transform}

Donoho\&candes presented a curvelet transform in 1999, It was very slow, and its method was developed by not using ridgetettransform, thus becoming fast and called fast discrete curvelet transform, the following steps show how can applying discrete curvelet transform [17,18].

1- Origin image split up in three sub bands.

2- Tiling is performed on sub bands $\Delta 1 \& \Delta 2$.

3- Discrete Ridge let transform is performed on tile.

Figure (1) shown performance of curve let transform.

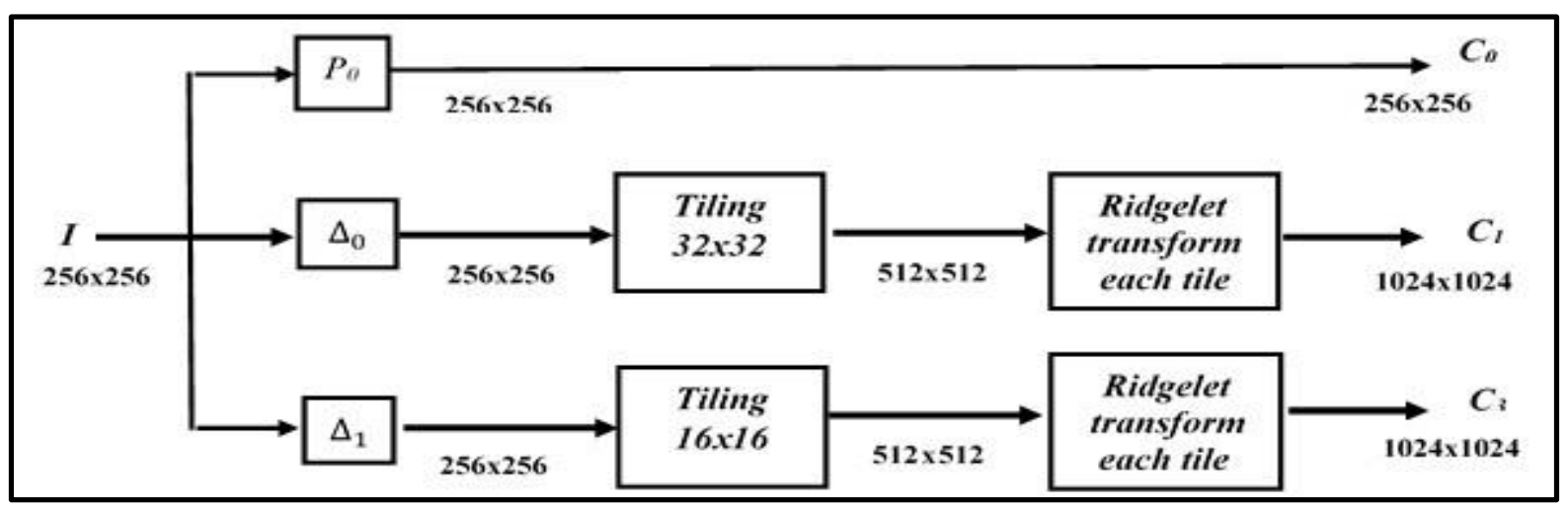

Figure 1. Curvelet transform

\section{Elman Neural Network}

Consists of three basic layers: the input layer, the extraction layer, and the hidden layer. The number of cells in the hidden layer depends on the difficulty and complexity of the question. This is a Feed-Backward Neural Network [19]. each input layer output is an input to each hidden layer cell. Either the output of the hidden layer is an input to the output layer as well as the input layer, figure (2) shown the Elman Neural Network structure [20]. 


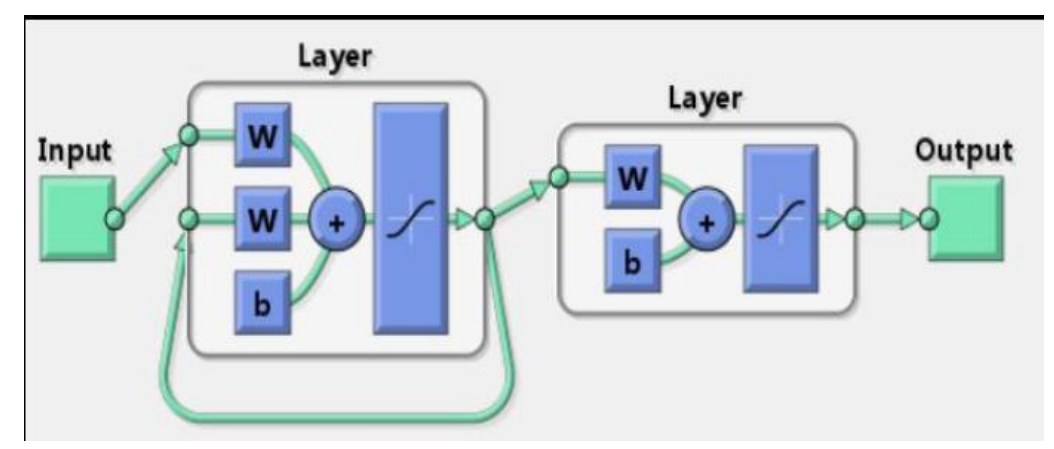

Figure 2. Elman neural network

\section{Color Space:}

There are many color systems that can represent the color digital image, and each of these systems have many characteristics that can be used in certain applications, the color system (RGB) is the most color systems used [21-22]. HSI consist thee layers: Hue, Saturation and Intensity the following equations using to convert RGB to HSI [23-25].

$h u e=\cos ^{-1}\left[\frac{R E D+0.5 * G R E E N-0.5 * B L U E}{\sqrt[2]{R E D^{2}+G R E E N^{2}+B L U E^{2}-R E B * G R E E N-R E D * B L U E-G R E E N * B L U E}}\right]$

saturation $=1-\left(\frac{M I N}{R E D+G R E E N+B L U E}\right)[M I N(R E D, G R E E N, B L U E]$

Intensity $=(R E D+G R E E N+B L U E) / 3$

\section{Proposal Algorithm}

The proposed algorithm steps will be displayed below to face recognition:

\section{Step One: Create Dataset}

1- read image for twenty students in computer science department of Tikrit university each one 25 images.

2- resize of image to $256 * 256$, remove noise.

\section{Step Two: Features Extraction}

1- decomposition of image using curve let transform to obtain the coefficient of transformations

2- using PCA on coefficients transformation to obtain features

\section{Step Three: Train Elman neural network}

1-Using only 18 images for each student and stay 3 for validation and 4 images to testing ..

2- the result of trained determine the optimal weights for the neural network.

\section{Step Four: Read image to recognition}

1 - read face new image in RGB color space

2- resize image to $256 * 256$.

3- covert color space from RGB to HSI

4- decomposition HSI to (hue, saturation, intensity);

5- selected saturation layer. 


\section{Step Five: Features Extraction from input image}

1- decomposition of input image using curve let transform to obtain the coefficient of transformations

2- using PCA on coefficients transformation to obtain features of input image

\section{Step Six: Test neural network}

1- enter the features of input image (the image is not trained on the network).

2- using optimal weights that obtained from network training

3- using Elman neural network

\section{Step Seven: Efficiency of the proposed method}

Number of correct face recognition $=\frac{n \text {. of correct recognition }}{\text { total number of enter image }}$

Figure (3) shown flowchart of steps of proposed algorithm

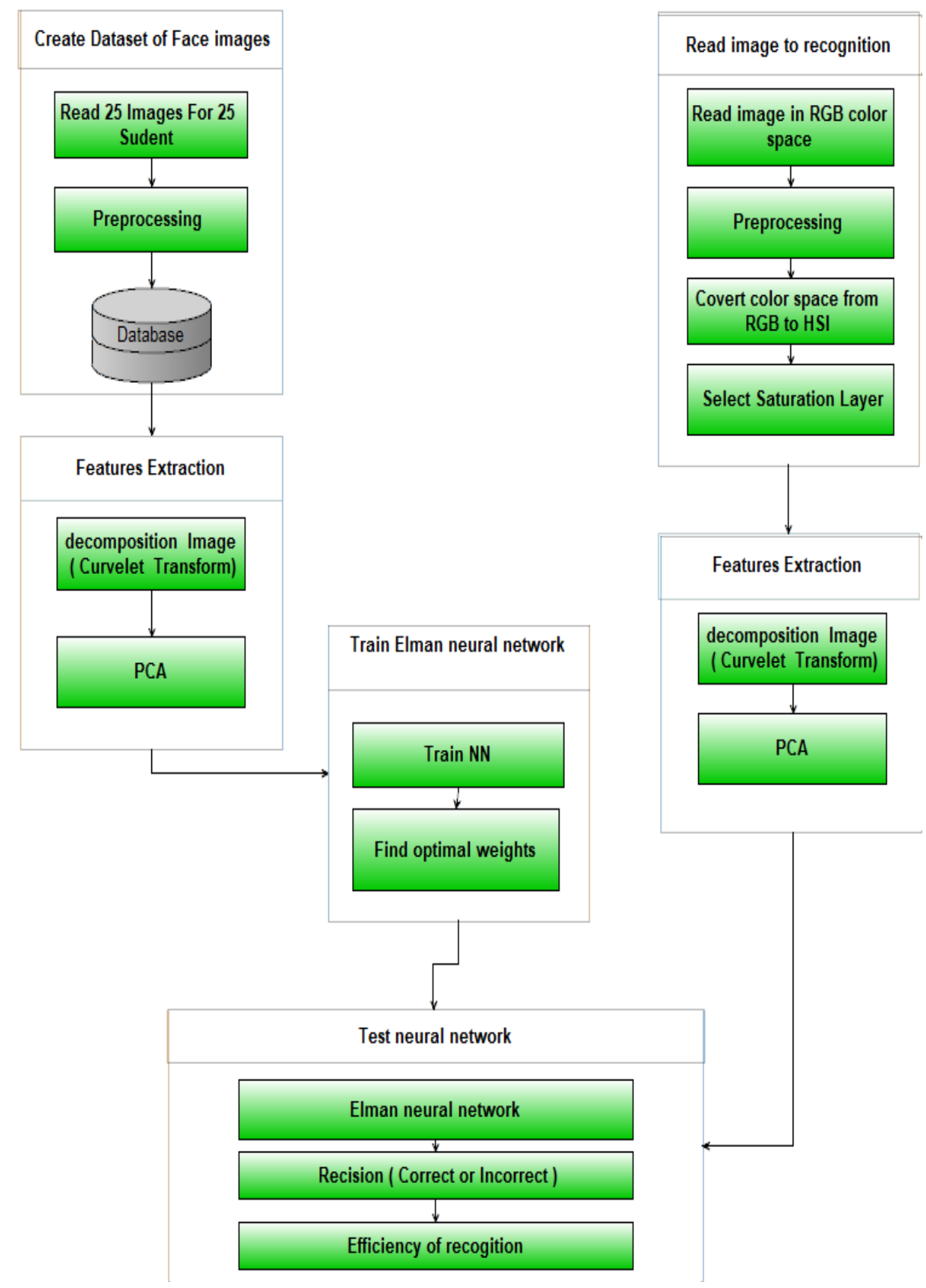

Figure 3. Proposed algorithm 


\section{Result and discussion}

The Neural Network is designed using four layers, the input layer consists of 120 nodes, output layer one node and two hidden layers first one consists 160 nodes and second one 120nodes, initial learning rate(ilr) $=0.17$.

In order to prove the improvement in face recognition processes, the algorithm was applied after different methods to compare with the proposed method.

\subsection{Face recognition using Elman and PCA:}

In this method using only PCA to features extraction and Elman to Recognition

\subsection{Face recognition using Elman and PCA with wavelet transform}

Wavelet transform used to obtain coefficients and PCA used to features extraction and last step using Elman NN.

\subsection{Proposed Algorithm}

After using this three method on20 images of different student each one test 20 images, table (1) and figure (4) show the result.

Table 1. Result of proposed algorithm

\begin{tabular}{|c|c|c|c|}
\hline & EIman \&\& PCA & WT,EIman,PCA & Proposed Algorithm \\
\hline Image & $\begin{array}{c}\text { images are not trained on } \\
\text { NN }\end{array}$ & images are not trained on $\mathrm{NN}$ & $\begin{array}{l}\text { images are not trained } \\
\text { on NN }\end{array}$ \\
\hline Im1 & $95 \%$ & $100 \%$ & $100 \%$ \\
\hline $\operatorname{Im} 2$ & $70 \%$ & $90 \%$ & $95 \%$ \\
\hline $\operatorname{Im} 3$ & $85 \%$ & $95 \%$ & $100 \%$ \\
\hline $\operatorname{Im} 4$ & $100 \%$ & $100 \%$ & $100 \%$ \\
\hline $\operatorname{Im5}$ & $55 \%$ & $75 \%$ & $90 \%$ \\
\hline $\operatorname{Im6}$ & $80 \%$ & $90 \%$ & $100 \%$ \\
\hline$\overline{\operatorname{Im} 7}$ & $70 \%$ & $75 \%$ & $85 \%$ \\
\hline $\operatorname{Im8}$ & $70 \%$ & $70 \%$ & $80 \%$ \\
\hline Im9 & $90 \%$ & $100 \%$ & $100 \%$ \\
\hline $\operatorname{Im10}$ & $40 \%$ & $55 \%$ & $75 \%$ \\
\hline Im11 & $100 \%$ & $100 \%$ & $100 \%$ \\
\hline $\operatorname{Im} 12$ & $85 \%$ & $95 \%$ & $100 \%$ \\
\hline Im13 & $45 \%$ & $65 \%$ & $70 \%$ \\
\hline $\operatorname{Im} 14$ & $85 \%$ & $85 \%$ & $95 \%$ \\
\hline Im15 & $100 \%$ & $100 \%$ & $100 \%$ \\
\hline Im16 & $80 \%$ & $85 \%$ & $100 \%$ \\
\hline $\operatorname{Im17}$ & $90 \%$ & $95 \%$ & $100 \%$ \\
\hline Im18 & $85 \%$ & $90 \%$ & $95 \%$ \\
\hline $\operatorname{Im} 19$ & $100 \%$ & $100 \%$ & $100 \%$ \\
\hline $\operatorname{Im} 20$ & $90 \%$ & $95 \%$ & $95 \%$ \\
\hline Efficiency & $81 \%$ & $88 \%$ & $94 \%$ \\
\hline
\end{tabular}




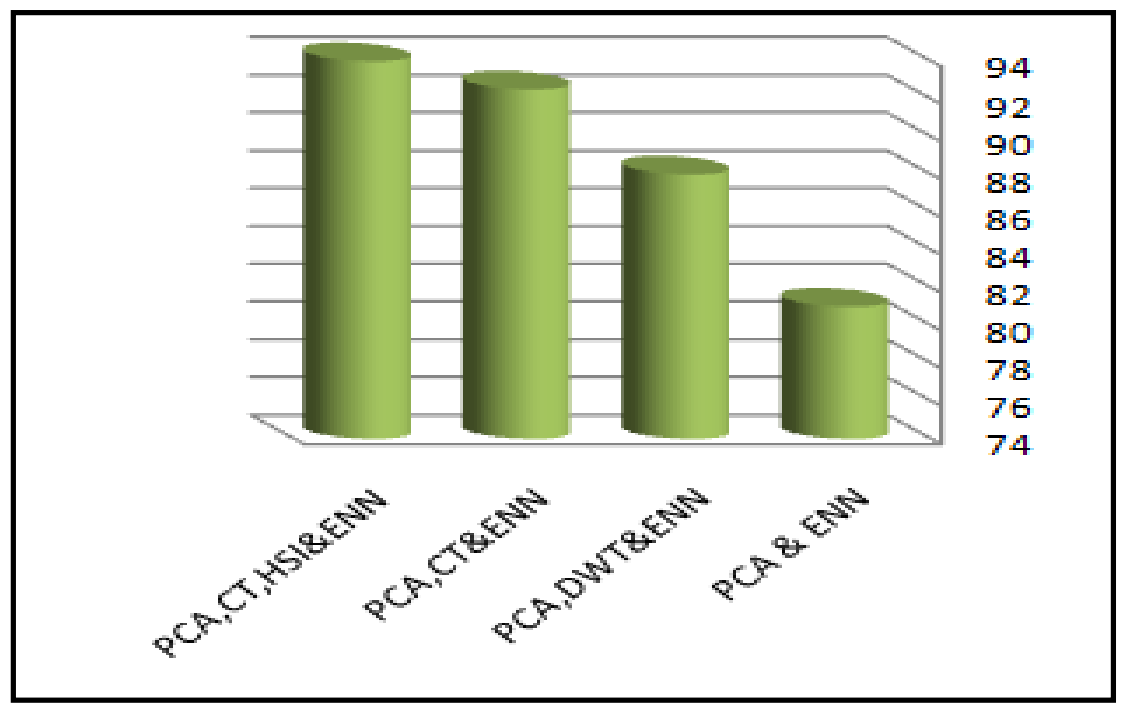

Figure 4. Result of proposed algorithm

The table shows that the percentage of discrimination adopted in the first method was $81 \%$. The second method was the percentage of discrimination $88 \%$. These methods are one of the many methods used in many researches that were explained in the second paragraph of the research. The proposed method has arrived 94\%. Figure (5) shown the difference in process is to distinguish each image individually.

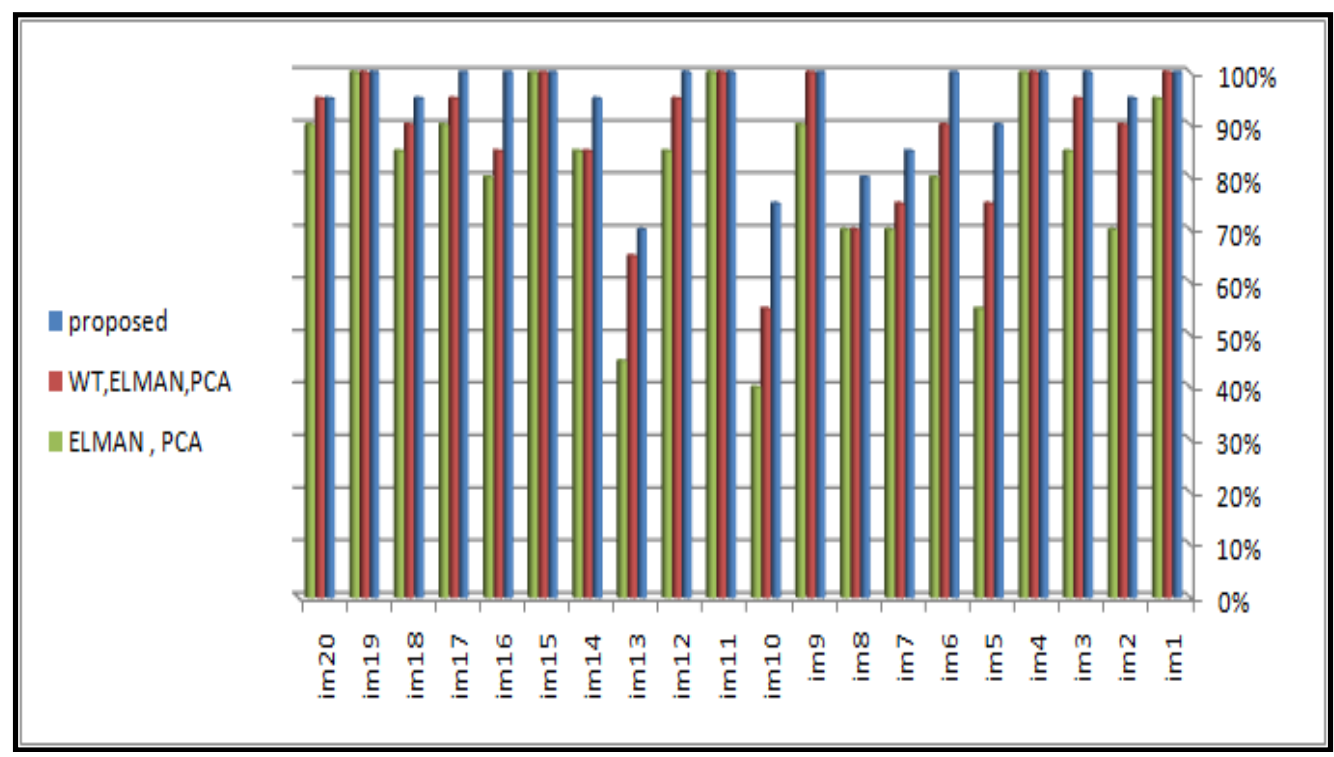

Figure 5. Recognition of individual image

It is clear from Figure (5) that the process of discrimination was measured in each picture showing that the proposed method was the best in all the images used in this research.

\section{8. conclusions}

Face recognition has become one of the most frequently used topics in many areas, including the use of an alternative to the password in opening some applications and other applications. the research presented a method to increase the efficiency of the Elman Neural Network using the curvelet transformations and some color properties. The results obtained indicate that the adoption of curvelet transformations provided better 
results than the use of wavelet transformations, the adoption of the HSI color scheme was better in terms of extracting the features increased the proportion of discrimination of the proposed method to $94 \%$.

\section{References}

[1]L. M. Dou, Z. L. Mu, Z. L. Li, A. Y. Cao, and S. Y. Gong, "Research progress of monitoring, forecasting, and prevention of rockburst in underground coal mining in China," International Journal of Coal Science \& Technology, vol. 1, no. 3, pp. 278-288, 2014.

[2]Tianwei Lan, Chaojun Fan, Sheng Li, Hongwei Zhang, and AndrianSergaevichBatugin, "Probabilistic Prediction of Mine Dynamic Disaster Risk Based on Multiple Factor Pattern Recognition," Advances in Civil Engineering, vol. 2018

[3]Kun Sun, Xin Yin, Mingxin Yang, Yang Wang, and Jianying Fan, "The Face Recognition Method Based on CSLBP and DBN," Mathematical Problems in Engineering, 2018,

[4]IssamDagher and Hussein Al-Bazzaz, "Improving the Component-Based Face Recognition Using Enhanced Viola-Jones and Weighted Voting Technique," Modelling and Simulation in Engineering, 2019.

[5]J. J. Richler, O. S. Cheung, and I. Gauthier, "Holistic processing predicts face recognition," Psychological Science, 2011.

[6]R.Shyam and Y. N. Singh, "A taxonomy of 2D and 3D face recognition methods," in Proceedings of the 1st International Conference on Signal Processing and Integrated Networks (SPIN '14), pp. 749-754, IEEE, Noida, India, February 2014

[7]RadheyShyam and Yogendra Narain Singh, "Multialgorithmic Frameworks for Human Face Recognition," Journal of Electrical and Computer Engineering, vol. 2016

[8]AliHussien Mary," Face Recognition Based Wavelet-PCA Features and Skin Color Model" Journal of Engineering and Development,2011.

[9]Maysaa Hameed Al-Hamdani," Face Image Recognition Using 2D PCA Algorithm" Eng. \& Tech. Journal,2012

[10] Abbas HussienMiry," Face Recognition Based Principal Component Analysis andWavelet Sub bands", Journal of Engineering and Development,2013

[11]Anmar Ali Mohammad,"Enhancethe PCA Method to Strength Face Recognition Systems" Iraq journal of information technology .2014.

[12]NashwanAlsalamAli," Face Recognition Using Stationary wavelet transform and Neural Network with Support Vector Machine ", Iraqi Journal of Science, 2015

[13] M. M. Mukhedkar and S. B. Powalkar, "Fast face recognition based on Wavelet Transform on PCA," International Conference on Energy Systems and Applications, Pune, 2016

[14]Fatma Zohra Chelali and Amar Djeradi, "Face Recognition Using MLP and RBF Neural Network with Gabor and Discrete Wavelet Transform Characterization: A Comparative Study," Mathematical Problems in Engineering, vol. 2015

[15]KanokmonRujirakul, Chakchai So-In, and BancharArnonkijpanich, "PEM-PCA: A Parallel ExpectationMaximization PCA Face Recognition Architecture,” The Scientific World Journal, vol. 2014

[16]IssamDagher and Hussein Al-Bazzaz, "Improving the Component-Based Face Recognition Using Enhanced Viola-Jones and Weighted Voting Technique," Modelling and Simulation in Engineering, vol. 2019 
[17]L.Boubchir and J. Fadili, "Multivariate statistical modelling of images with the curvelet transform," in Proceedings of the 8th International Conference on Signal Processing, Pattern Recognition, and Applications, pp. 747-750, 2005

[18].Khalil I. Alsaif and Esraa S. Hussein," Curvelet Transform for Kidney Stones Detection", International Journal of Computer Science and Information Security, Vol. 15, No. 1, 2017.pp335-341

[19]Lei Sun, Wenjun Yi, Dandan Yuan, and Jun Guan, "Application of Elman Neural Network Based on Genetic Algorithm in Initial Alignment of SINS for Guided Projectile," Mathematical Problems in Engineering, vol. 2019 ,

[20]Jun Pi, Jiangbo Huang, and Long Ma, "Aeroengine Fault Diagnosis Using Optimized Elman Neural Network," Mathematical Problems in Engineering, vol. 2017

[21]HayrettinToylan and HilmiKuscu, "A Real-Time Apple Grading System Using Multicolor Space," The Scientific World Journal, vol. 2014, pp1-12

[22]Zhenmin Zhu, Ruichao Song, Hui Luo, Jun Xu, and Shiming Chen, "Color Calibration for Colorized Vision System with Digital Sensor and LED Array Illuminator," Active and Passive Electronic Components, vol. 2016, pp1-16

[23] R. Palalic, Durakovic, B., Brankovic, A., and Ridic, O., "Students' Entrepreneurial Orientation Intention, Business Environment, and Networking: Insights from Bosnia and Herzegovina", Students' Entrepreneurial Orientation Intention, Business Environment, and Networking: Insights from Bosnia and Herzegovina, vol. 11, no. 4, pp. 240-255, 2016. 